\title{
The impact of aesthetic evaluation and physical ability on dance perception
}

\author{
Emily S. Cross ${ }^{1,2,3}{ }^{*}$, Louise Kirsch ${ }^{1}$, Luca F. Ticini ${ }^{1,4}$ and Simone Schütz-Bosbach ${ }^{1}$ \\ 1 Junior Research Group "Body and Self," Max Planck Institute for Human Cognitive and Brain Sciences, Leipzig, Germany \\ ${ }^{2}$ Behavioural Science Institute, Donders Institute for Brain, Cognition and Behaviour, Radboud University Nijmegen, Netherlands \\ ${ }^{3}$ School of Psychology, Bangor University, Wales, UK \\ ${ }^{4}$ Italian Society of Neuroaesthetics "Semir Zeki," Trieste, Italy
}

Edited by:

Idan Segev, The Hebrew University of Jerusalem, Israel

\section{Reviewed by:}

Marcel Brass, Ghent University,

Belgium

Tamer Demiralp, Istanbul University,

Turkey

\section{*Correspondence:}

Emily S. Cross, School of Psychology,

Adeilad Brigantia, Bangor University,

Bangor, Wales LL57 2AS, UK.

e-mail: e.cross@psych.ru.nl
The field of neuroaesthetics attracts attention from neuroscientists and artists interested in the neural underpinnings of esthetic experience. Though less studied than the neuroaesthetics of visual art, dance neuroaesthetics is a particularly rich subfield to explore, as it is informed not only by research on the neurobiology of aesthetics, but also by an extensive literature on how action experience shapes perception. Moreover, it is ideally suited to explore the embodied simulation account of esthetic experience, which posits that activation within sensorimotor areas of the brain, known as the action observation network (AON), is a critical element of the esthetic response. In the present study, we address how observers' esthetic evaluation of dance is related to their perceived physical ability to reproduce the movements they watch. Participants underwent functional magnetic resonance imaging while evaluating how much they liked and how well they thought they could physically replicate a range of dance movements performed by professional ballet dancers. We used parametric analyses to evaluate brain regions that tracked with degree of liking and perceived physical ability. The findings reveal strongest activation of occipitotemporal and parietal portions of the AON when participants view movements they rate as both esthetically pleasing and difficult to reproduce. As such, these findings begin to illuminate how the embodied simulation account of esthetic experience might apply to watching dance, and provide preliminary evidence as to why some people find enjoyment in an evening at the ballet.

\section{Keywords: dance, neuroaesthetics, parietal, visual, fMRI, AON, ballet}

\section{INTRODUCTION}

In recent years, the nascent field of neuroaesthetics has gained momentum as scientists interested in the neural processes underlying an esthetic experience, such as a beautiful painting, piece of music, or dance performance, have begun to elucidate the links between sensory input and the observers' affective evaluation (Zeki, 1999; Blood and Zatorre, 2001; Cela-Conde et al., 2004; Kawabata and Zeki, 2004). Most neuroaesthetics research to date has focused on brain engagement when participants evaluate paintings or music (for reviews, see Di Dio and Gallese, 2009; Chatterjee, 2011). One theory emerging from the neuroaesthetics research on visual art is that an important factor in shaping an observer's esthetic experience is the simulation of actions, emotions, and corporeal sensations visible or implied in an artwork (Freedberg and Gallese, 2007). Freedberg and Gallese (2007) suggest that embodied resonance of art in an observer can be driven by the content of the work (such as empathic pain experienced when viewing the mangled bodies in Goya's Que hay que hacer mas) or by the visible traces of the artists' creation (such as evidence for vigorous handling of the artistic medium, like that which might be experienced when viewing a Jackson Pollock painting). While an embodied simulation account of esthetic experience provides a useful context for considering an observer's esthetic experience of art, the authors acknowledge that "a question arises about the degree to which empathic responses to actions in real life differ from responses to actions that are represented in paintings and sculpture" (p. 202). In the present study, we address this question by studying an artistic medium where the actions required to create the artwork are the artwork. Specifically, we investigate the relationship between esthetic experience, physical ability, and activation of sensorimotor brain regions when watching dance.

Compared with the abundance of studies focused on music and visual art, the neuroaesthetics of watching dance has received relatively limited research attention (Calvo-Merino et al., 2008, 2010; Hagendoorn, 2010; Cross and Ticini, 2011). Dance neuroaesthetics is a particularly rich topic to investigate, as it is informed not only by research on the neural substrates of esthetic experience, but also by an extensive literature on how the experience of action shapes action perception (e.g., Decety and Grezes, 1999; Buccino et al., 2001; Casile and Giese, 2006; Aglioti et al., 2008), including a number of studies specifically looking at dance perception among dance experts (Calvo-Merino et al., 2005, 2006; Cross et al., 2006) and novices (Cross et al., 2009a,b).

By now, numerous studies have demonstrated overlap between action perception and performance in the human motor system. Supporting evidence is provided by experiments measuring 
corticospinal excitability with motor evoked potentials (MEPs; e.g., Fadiga et al., 1995) and changes in blood oxygenation level dependent $(\mathrm{BOLD})$ responses in motor areas of the brain with functional magnetic resonance imaging (fMRI; e.g., Grafton et al., 1996; Grèzes and Decety, 2001; Caspers et al., 2010; Molenberghs et al., in press). Of particular interest in these studies are brain regions that respond when watching others move, collectively known as the action observation network (AON; Grèzes and Decety, 2001; Cross et al., 2009b; Gazzola and Keysers, 2009). This network, comprising premotor, parietal, and occipitotemporal cortices, is believed to help us make sense of others' bodies in motion, in order to help us decode the goals and intentions underlying their movements (Gallese et al., 2004; Rizzolatti and Sinigaglia, 2010).

A noteworthy approach for investigating how the AON subserves action perception is to measure how an observer's prior physical or visual experience influences his or her perception of others' actions. Scientists from a growing number of laboratories are turning to expert and novice dancers to help address such questions (Calvo-Merino et al., 2005, 2006; Cross et al., 2006, 2009b; Bläsing et al., 2010). One consistent finding this research has revealed is that when dancers observe a type of style of movement that they are physically adept at performing, greater activity is recorded within parietal and premotor portions of the AON (e.g., Calvo-Merino et al., 2005, 2006; Cross et al., 2006, 2009a,b). Moreover, it has also been demonstrated that the amplitude of the response within parietal and premotor portions of the $\mathrm{AON}$, as measured by fMRI, increases parametrically the better an observer is able to perform the observed dance sequence (Cross et al., 2006).

Such research has opened a gateway to understanding how specific neural changes are associated with an individual's ability to perform highly complex and coordinated actions. However, findings in this vein stop short at being able to explain how and why dance observers often derive intense pleasure from watching dance (Cross and Ticini, 2011). Is it because we embody the forms and movements articulated by the dancers within our own motor system, consistent with the embodied simulation account of esthetic experience (Freedberg and Gallese, 2007), or does enjoyment stem from a more purely visual experience? To our knowledge, only one published study (Calvo-Merino et al., 2008) has explored how participants' subjective evaluations of dynamic displays of dance correlate with activity within sensorimotor brain regions that compose the AON. In this study, the authors asked dance-naïve participants to carefully observe a number of videos featuring different dance movements while undergoing fMRI (Calvo-Merino et al., 2008). Approximately 1 year later, participants watched the dance videos again, and this time their task was to rate each video using a five-point Likert scale on the five key esthetic dimensions identified by Berlyne (1974): like-dislike, simple-complex, dull-interesting, tense-relaxed, and weak-powerful. The authors averaged participants' responses and focused on how the consensus ratings for each dance stimulus related to brain responses. They found that when participants watched dance movements they rated as highly likable, increased activity emerged within right premotor cortex, as well as bilateral early visual regions. The authors concluded that the premotor portion of the AON might thus be important in assigning an automatic and implicit esthetic evaluation to dance.

This previous study offers an intriguing first glimpse of the neural substrates that might underlie the esthetic experience of watching dance. However, it also leaves many enticing questions open for further exploration. For example, since Calvo-Merino et al. (2008) explicitly chose to focus on the brain responses corresponding to a group's consensus esthetic evaluation of each stimulus, it remains unknown how individual ratings of a dance's esthetic value might be related to AON activity. We know from prior work that parietal and premotor portions of the AON are sensitive to individuals' physical experience with movements (e.g., Calvo-Merino et al., 2005; Cross et al., 2006), and that responses within visual and premotor regions correlate with how much a group likes watching certain movements (Calvo-Merino et al., 2008), but these how two factors interact remains unknown. In the present study, we aim to address this interaction between physical ability and esthetic evaluation.

We selected participants with little experience performing or watching dance and asked them to observe videos depicting movements performed by expert ballet dancers. Following each video, participants rated either how much they liked watching the movement, how well they could physically reproduce each movement, or responded to a factual question concerning the content of the video (such as whether the dancer jumped or not). Because CalvoMerino et al. (2008) found BOLD response correlations only with participants' like-dislike ratings (and not the other four esthetic dimensions identified by Berlyne (1974), we focus on only the like-dislike esthetic dimension in this study.

We analyzed the imaging data using participants' individual liking and physical ability ratings as parametric modulators via three main contrasts. The first evaluated regions modulated by how much participants liked a movement. If individual ratings are largely consistent with the group-averaged ratings used by Calvo-Merino et al. (2008), then we should find increased activation of right premotor and early visual cortices when participants watched movements they liked. The second contrast replicates Cross et al. (2006), who measured regions parametrically modulated by participants' perceived ability to perform each movement. If such ratings made by expert dancers generalize to ratings made by non-dancers, then we might expect left parietal and premotor cortices to show increased activity as participants rate actions as increasingly easy to replicate. The third contrast evaluates the interaction between liking and perceived ability, while a related behavioral analysis enables us to measure whether a relationship emerges between subjective ratings of these two modulators. Findings should further our understanding of the embodied simulation account of esthetic experience as it may apply to dance.

\section{MATERIALS AND METHODS PARTICIPANTS}

Twenty-two physically and neurologically healthy young adults were recruited from the fMRI Database of the Max Planck Institute for Human Cognitive and Brain Sciences (Leipzig, Germany). All were monetarily compensated for their involvement, and gave written informed consent. The local ethics committee approved all components of this study. The 22 participants ( 9 females) ranged 
in age from 21 to 33 years (mean $=24.8$ years, $S D=2.9$ years). All participants were strongly right handed as measured by the Edinburgh Handedness Inventory (Oldfield, 1971).

Moreover, all participants were recruited as naïve observers with limited or no dance experience, qualified by completion of a questionnaire following the experimental manipulation to evaluate past experience in performing and watching dance. No participant had formal training in ballet or modern dance (though some participants took one semester of ballroom dance training in school, as is required in some regions in Germany). When asked to evaluate their ability as a dancer on a 1 - to 5 -scale ( $1=$ awful; $2=$ bad; $3=$ intermediate; $4=$ good; $5=$ very good), participants scored themselves with a mean rating of $2.7(\mathrm{SD}=1.12)$. To quantify experience with dance observation, the mean number of professional dance performances (or theatre/opera performances that had some dance element) attended each year by participants was $1.02(\mathrm{SD}=1.06)$.

\section{STIMULI AND DESIGN}

Stimuli featured a male or female dancer performing a dance movement. The dancers, both members of the Leipziger Ballett performed a range of movements varying in complexity, speed, difficulty, and size, as well as to use movement from both classical and contemporary dance vocabularies. From the footage captured of both dancers, 64 different dance video stimuli were constructed, each $3 \mathrm{~s}$ in length. To establish a stimulus-specific baseline, two additional $3 \mathrm{~s}$ videos were used, created from footage of each dancer standing still in a neutral posture in the same studio setting.

\section{MOTION ENERGY OUANTIFICATION}

Because each dance sequence differed in terms of the size, speed, and spatial range of the movements, we took an additional step to attempt to control for such differences in the imaging data. In order to do this, we quantified the motion energy in each video clip using a custom Matlab algorithm, based on motion recognition work by (Bobick, 1997) in computer science. Such quantification of motion energy has been applied successfully before to stimuli used in neuroimaging studies of action observation (Schippers et al., 2010; Cross et al., in press-a). With our particular algorithm, we converted each movie to gray-scale, and then calculated a difference image for pairs of consecutive frames in each movie. The difference image was thresholded so that any pixel with more than 10 units luminance change was classified as "moving." The average numbers of moving pixels per frame and per movie were summed to give a motion energy score for that movie.

\section{fMRI TASK}

During functional neuroimaging, all videos were presented via Psychophysics Toolbox 3 running under Matlab 7.2. The videos were presented in full color with a resolution of $480 \times 270$ pixels using a back projection system, which incorporated a LCD projector that projected onto a screen placed behind the magnet. The screen was reflected on a mirror installed above participants' eyes. Participants completed one functional run $34 \mathrm{~min}$ in duration, comprising 128 experimental trials (2 presentations of each of the 64 dance videos) organized randomly. Each experimental trial video was followed by one of the two main questions of interest (how much did you like it?/how well could you reproduce it?); participants' task was to watch each video closely and answer the question following the video. Importantly, trials were arranged to collect one liking and one reproducibility rating for each stimulus, thus participants never answered the same question about a particular video twice. In order to reduce task predictability and to encourage the maintenance of focus throughout the experiment, eight additional trials were randomly interspersed among the experimental trials, after each of which participants were asked an unpredictable yes-no question about the video content, addressing various features of the stimulus movement (e.g., did the dancer jump?; did the dancer turn?; did the dancer's hands touch the ground?). Also interspersed randomly across the 128 experimental trials were 16 repetitions ( 8 trials with each of the 2 dancers) of the 3-s videos of the dancers standing still in a neutral position. The intertrial intervals were pseudologarithmically distributed between 4 and $8 \mathrm{~s}$. A schematic depiction of the task is illustrated in Figure 1.

\section{fMRI DATA ACQUISITION}

All data were collected at the Max Planck Institute for Human Cognitive and Brain Sciences (Leipzig, Germany). Functional images were acquired on a Bruker 3-T Medspec 20/100 whole-body MR scanning system, equipped with a standard birdcage head coil. Functional images were acquired continuously with a single shot gradient echo-planar imaging (EPI) sequence with the following parameters: echo time $\mathrm{TE}=30 \mathrm{~ms}$, flip angle $90^{\circ}$, repetition time TR $=2,000 \mathrm{~ms}$, acquisition bandwidth $100 \mathrm{kHz}$. Twenty-four axial slices allowing for full-brain coverage were acquired in ascending order (pixel matrix $=64 \times 64$, FOV $=24 \mathrm{~cm}$, resulting

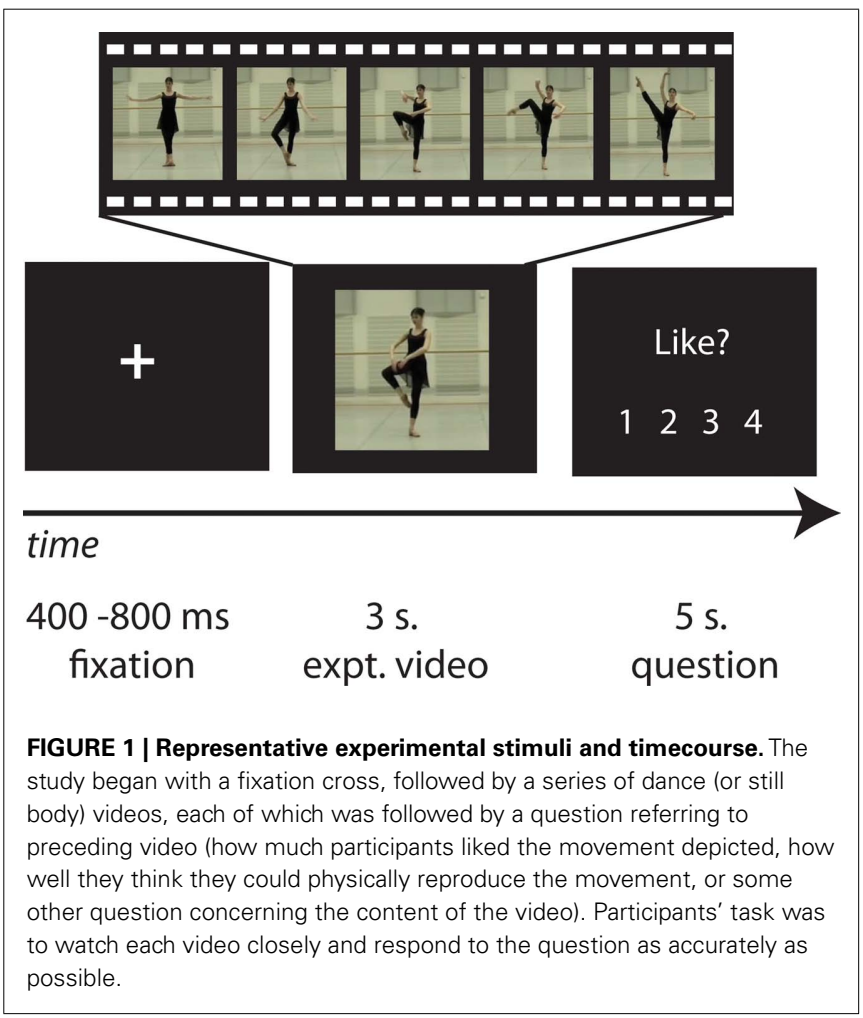


in an in-plane resolution of $3.75 \mathrm{~mm} \times 3.75 \mathrm{~mm}$, slice thickness $=4 \mathrm{~mm}$, interslice gap $=1 \mathrm{~mm})$. Slices were oriented parallel to the bicommissural plane (AC-PC line). The first two volumes of each functional run were discarded to allow for longitudinal magnetization to approach equilibrium, and then an additional 1015 volumes of axial images were collected.

Geometric distortions were characterized by a B0 field-map scan [consisting of a gradient echo readout (32 echoes, interecho time $0.64 \mathrm{~ms}$ ) with a standard 2D phase encoding]. The B0 field was obtained by a linear fit to the unwarped phases of all odd echoes. Prior to the functional run, 24 two-dimensional anatomical images $(256 \times 256$ pixel matrix, T1-weighted MDEFT sequence) were obtained for normalization purposes. In addition, for each subject a sagittal T1-weighted high-resolution anatomical scan was recorded in a separate session on a different scanner (3-T Siemens Trio, 160 slices, $1 \mathrm{~mm}$ thickness). The anatomical images were used to align the functional data slices with a $3 \mathrm{D}$ stereotaxic coordinate reference system.

\section{fMRI DATA ANALYSIS}

Data were realigned, unwarped, corrected for slice timing, normalized to individual participants' T1-segmented anatomical scans with a resolution of $3 \mathrm{~mm} \times 3 \mathrm{~mm} \times 3 \mathrm{~mm}$, and spatially smoothed $(8 \mathrm{~mm})$ using SPM8 software. A design matrix was fitted for each participant, with each $3 \mathrm{~s}$ dance movie trial modeled by a boxcar with the duration of the video convolved with the standard hemodynamic response function. Three additional parametric modulators were included for the main dance video trials: participants' individual ratings of how much they liked each dance sequence, participants' individual ratings of how well they thought they could reproduce each dance sequence, and a regressor expressing the mean motion energy of each video, which compensates for major differences in contrasts of interest due to varying amounts of movement between stimuli (Cross et al., in press-a). Additional regressors in the model included the "still body baseline" (comprising the 16 still body videos), the "test questions" (comprising the eight trials where participants were asked a yes-no question about the previously viewed video), and the "question and response phase" (encompassing the time when participants were asked each question and made a keypress response).

Imaging analyses were designed to achieve four objectives. The first group-level analysis evaluated which brain regions were more active when observing a dancer's body in motion compared to viewing a dancer's body standing still. Such a contrast enables the localization of brain regions responsive to dance per se, and not extraneous features of the display that are not of interest for this study (e.g., the dancers' identity, the layout of the dance studio, etc.). Regions that emerged from this contrast, illustrated in Figure 2; were used to create a task-specific mask for all subsequent analyses reported in the paper, at the $p<0.001, k=10$ voxel level. The second analysis identified brain regions responsive to esthetic appraisal of dance movements. To accomplish this, we evaluated both directions of the parametric regressor for "liking," to differentiate between brain regions showing an increased response with increased liking and those showing an increased response with decreased liking. The third analysis followed the identical

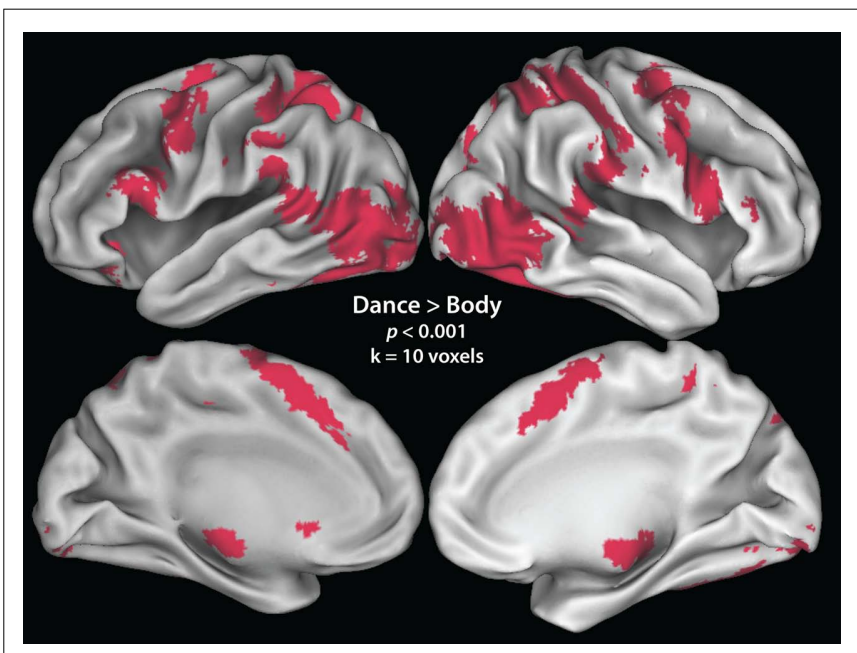

FIGURE 2 | Neural regions active in the contrast comparing all dance observation > static body baseline. This contrast was made to determine, in an unbiased, subject- and task-specific manner, which regions were to be included in the mask of the AON.

approach for the parametric modulator for "perceived physical ability." The fourth analysis evaluated the interaction between "liking" and "perceived physical ability." Two directions of the interaction were evaluated, highlighting in one direction regions that responded more when participants liked a movement but perceived it as difficult to reproduce, and in the other direction brain regions that were more active when participants watched movements they did not like but perceived as easy to reproduce. All contrasts were evaluated at $p_{\mathrm{u}}<0.001$ (uncorrected for multiple comparisons), and $k=10$ voxels. For the main parametric contrasts, we focus on those results that reached a cluster-level significance of $p_{c o r} .<0.05$ (FDR-corrected for multiple comparisons) ${ }^{1}$. For anatomical localizations, all functional data were referenced to cytoarchitectonic maps using the SPM Anatomy Toolbox v1.7 (Eickhoff et al., 2005, 2006, 2007). For visualization purposes, the $t$-image of the AON mask is displayed on partially inflated cortical surfaces using the PALS data set and Caret visualization tools (Figure 2; http://brainmap.wustl.edu/caret). All other analyses are illustrated on an averaged high-resolution anatomical image of the study population (Figures 3 and 4 ).

\section{RESULTS}

The first imaging analysis, evaluated as all dance $>$ still bodies, revealed broad activation in a network comprising areas classically associated with action observation (e.g., Grèzes and Decety, 2001; Cross et al., 2009b; Caspers et al., 2010; Grosbras et al., in press), including bilateral parietal, premotor, supplemental motor, and occipitotemporal cortices. A full listing of regions can be found in Table 1. This contrast, illustrated in Figure 2; was used as a mask for all analyses described below.

${ }^{1}$ For completeness and transparency, the tables list all regions significant at the uncorrected threshold of $p<0.001$. 

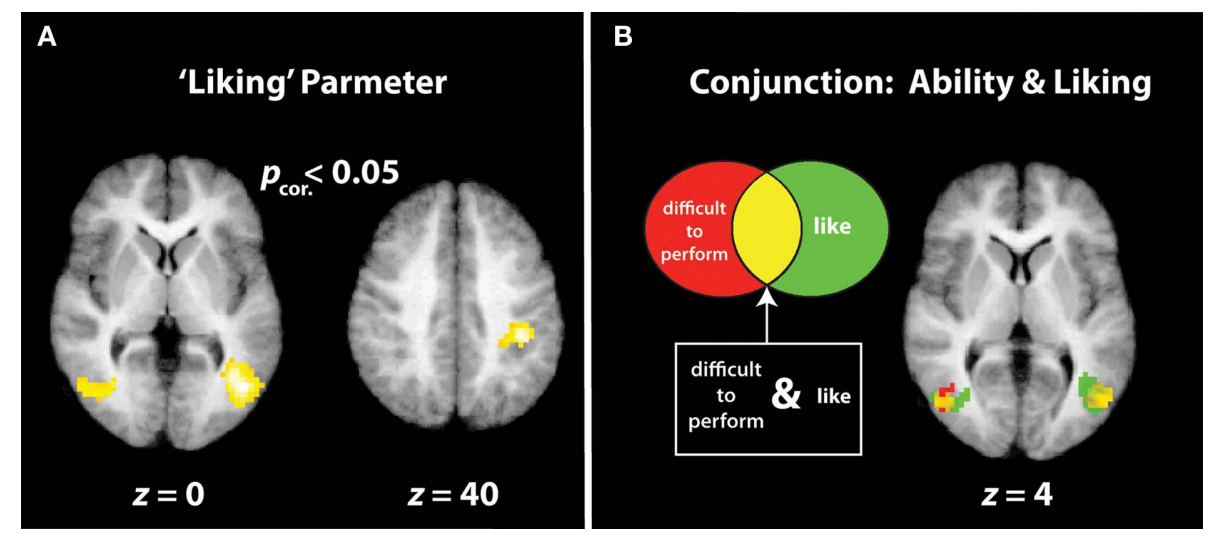

FIGURE 3 | "Increased liking" and "decreased physical ability" parameters. (A) Illustrates the three cluster-corrected activations that demonstrate increasing BOLD signal strength the more participants like the dance movement. (B) Illustrates the conjunction between regions with greater responses the more difficult participants think a

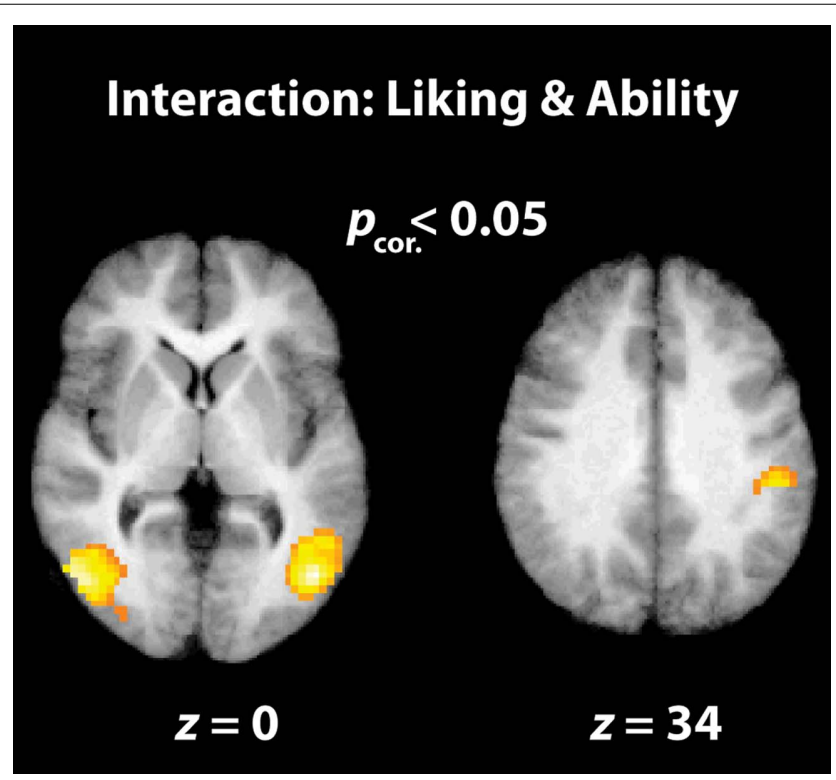

FIGURE 4 | Interaction between "liking" and "physical ability" parameters. The parietal and visual brain regions illustrated here are cluster-corrected activations that are active when participants watch dance movements that they rate as being highly enjoyable to watch, but very difficult to reproduce.

\section{AON REGIONS MODULATED BY LIKING}

The positive direction of this parametric contrast revealed bilateral activation within visual brain regions implicated in the processing of complex motion patterns (namely, area $\mathrm{V} 5 / \mathrm{MT}+$ ), and human bodies (ITG/MTG), as well as a large cluster within the right inferior parietal lobule (IPL; Figure 3A; Table 2A). The inverse direction of this contrast, which interrogated regions showing an increased BOLD response the less participants liked a movement, did not reveal any suprathreshold activations.

movement would be to reproduce (activations in red) and regions that are more active the more participants like an observed movement [same activations as those illustrated in (A); in green]. Voxels of overlap between the two parametric contrasts are illustrated in yellow.

\section{AON REGIONS MODULATED BY PERCEIVED PERFORMANCE ABILITY}

In direct contrast to the results reported previously with expert dancers (Cross et al., 2006), no suprathreshold activations emerged from the positive direction of the analysis that evaluated brain regions that increase in response the better a participant thinks he or she can perform an observed movement, either at the corrected or uncorrected level. The inverse contrast, which evaluated brain regions that became increasingly active the less participants thought they could perform the observed movement, resulted in no activations reaching cluster-corrected significance, though several uncorrected clusters emerged within bilateral middle occipital gyri (Table 2B). For comparison of the visual regions activated by liking and perceived difficulty to reproduce an observed movement, Figure 3B illustrates the overlap of both parametric contrasts. As Figure 3B shows, similar portions of the middle temporal gyri are engaged both by movements that participants enjoy watching and by those they believe are difficult to reproduce. This strongly suggests that these two factors are not independent, an issue to which we return in greater detail below. Even when the effects of liking and perceived physical ability were evaluated at the whole brain level (i.e., not masked by the dance $>$ body contrast), no additional regions emerged.

\section{INTERACTION BETWEEN LIKING AND PHYSICAL ABILITY}

The final analysis examined the interaction between liking and perceived ability when watching dance. The behavioral data indicate that liking and physical ability ratings were not entirely independent; in other words, participants liked more those movements they rated as difficult to perform. Pearson correlation coefficients calculated on an individual subject level demonstrate that the relationship between liking and physical ability ranged from $r=0.021$ to $r=-0.615$, with an average $r=-0.27(\mathrm{SD}=0.21)$. The presence of an interaction between these variables in the behavioral data enables us to investigate brain regions showing an increased BOLD signal when watching movements that are increasingly enjoyable to watch and increasingly difficult to execute. This 
Table 1 | Main effect of observing dance compared to a still body.

\begin{tabular}{|c|c|c|c|c|c|c|}
\hline \multirow[t]{2}{*}{ Anatomical brain regions } & \multirow[t]{2}{*}{ BA } & \multicolumn{3}{|c|}{ MNI coordinates } & \multirow[t]{2}{*}{ Functional name } & \multirow[t]{2}{*}{$T$-score } \\
\hline & & $x$ & $y$ & $z$ & & \\
\hline R inferior parietal lobule & 2 & 30 & -46 & 52 & IPL & 8.84 \\
\hline R fusiform gyrus & 37 & 42 & -49 & -17 & & 8.78 \\
\hline L middle occipital gyrus & $39 / 19$ & -45 & -73 & 1 & MOG & 12.84 \\
\hline L fusiform gyrus & 37 & -42 & -52 & -23 & & 8.71 \\
\hline$R$ thalamus & & 6 & -31 & -5 & & 8.42 \\
\hline$L$ thalamus & & -3 & -31 & -5 & & 7.72 \\
\hline Midline midbrain & & 0 & -22 & -23 & & 4.73 \\
\hline R precentral gyrus & $4 / 6$ & 33 & -7 & 49 & PMd & 7.83 \\
\hline L precentral gyrus & $4 / 6$ & -30 & -7 & 49 & PMd & 7.55 \\
\hline L inferior frontal gyrus & 44 & -45 & 20 & -2 & PMv & 3.59 \\
\hline L posterior cingulate gyrus & $19 / 30$ & -21 & -43 & 19 & & 4.77 \\
\hline $\mathrm{R}$ posterior cingulate gyrus & $19 / 30$ & 27 & -46 & 16 & & 4.75 \\
\hline R inferior frontal gyrus & 45 & 48 & 29 & 16 & & 4.45 \\
\hline L superior parietal lobule & 31 & -15 & -28 & 40 & SPL & 4.43 \\
\hline L caudate nucleus & & -3 & 11 & 1 & & 3.90 \\
\hline
\end{tabular}

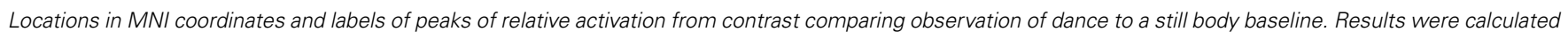
at $p_{\text {uncorrected }}<0.001, k=10$ voxels. Up to three local maxima are listed when a cluster has multiple peaks more than 8 mm apart. Entries in bold denote activations significant at the FDR cluster-corrected level of $p<0.05$. Abbreviations for brain regions: BA, Brodmann's area; $R$, right; $L$, left; $M T G$, middle temporal gyrus; PMd, dorsal premotor cortex; SPL, superior parietal lobule.

analysis revealed activity within bilateral occipitotemporal cortices and the right IPL (Figure 4; Table 2C). It is of note that broader AON activation emerges in the uncorrected results (Table 2C), including left parietal and right premotor cortices. The inverse interaction, examining brain regions responding to movements participants dislike but can perform, revealed no suprathreshold activations at corrected or uncorrected levels.

\section{DISCUSSION}

The present study represents the first attempt to investigate the relationship between esthetic appreciation and observers' physical ability when watching dance. Dance-naïve participants watched a series of videos featuring expert dancers and were asked to make explicit judgments about each video, including how much they liked the movements and how well they believed they could execute them. We report two novel findings that have the potential to inform our understanding of how we perceive the art of dance. First, our behavioral data indicate that participants tended to like movements more that they perceived as difficult to physically perform. Second, we report that the interaction between liking and physical ability is represented within occipitotemporal and parietal regions of the AON. We consider now how these findings inform our understanding of the embodied simulation account of esthetic experience, as well as the relevance of the present data to prior work on expertise and aesthetics. We conclude with consideration of possible future directions for dance neuroaesthetics.

\section{LIKING WHAT WE CANNOT DO}

In the present study, participants reported liking dance movements more that they perceived as difficult to perform themselves. Anecdotally, this finding resonates with the fact that spectators routinely pay high prices to watch the outstanding physical mastery of acrobats in Cirque du Soleil, slam-dunking basketball players in an NBA game, or the exacting precision of the Bolshoi corps de ballet. If every audience member could reproduce the movements made by the acrobats, athletes or dancers, then such events would no longer be spectacular. One possible account of this relationship could be that the seemingly effortless nature with which highly physically skilled individuals perform difficult and spectacular movements leads to increased liking precisely because the spectator knows she is witnessing a physical feat well beyond her own abilities.

A stronger preference for movements that appear easy for the dancer, but difficult for the observer to perform could possibly inform a perceptual fluency account of why we rate certain stimuli as more likable than others (Berlyne, 1974). A number of studies demonstrate that people tend to like stimuli more that are easy to understand (e. g., Jacoby and Dallas, 1981; Whittlesea, 1993). Researchers have also demonstrated that we like objects more that we have watched others interact with smoothly and efficiently, compared to objects that were interacted with awkwardly (Hayes et al., 2008), thus demonstrating a link between liking and perceived action fluidity. In the present study, we add another 
Table 2 | Parametric effects of and interaction between liking and physical ability.

\begin{tabular}{|c|c|c|c|c|c|c|}
\hline \multirow[t]{2}{*}{ Anatomical brain regions } & \multirow[t]{2}{*}{ BA } & \multicolumn{3}{|c|}{ MNI coordinates } & \multirow[t]{2}{*}{ Functional name } & \multirow[t]{2}{*}{$T$-score } \\
\hline & & $x$ & $y$ & $z$ & & \\
\hline R middle temporal gyrus & 19 & 45 & -67 & 4 & V5/MT+ & 5.92 \\
\hline $\mathbf{R}$ inferior temporal gyrus & 19 & 45 & -73 & -8 & ITG & 5.68 \\
\hline $\mathbf{R}$ inferior temporal gyrus & 37 & 51 & -61 & -11 & ITG & 4.69 \\
\hline L middle temporal gyrus & $19 / 39$ & -33 & -67 & 1 & MTG & 4.61 \\
\hline L middle occipital gyrus & 39 & -45 & -76 & 13 & MOG & 4.20 \\
\hline R supramarginal gyrus & 2 & 39 & -34 & 40 & IPL & 5.75 \\
\hline R inferior parietal lobule & $40 / 41$ & 45 & -31 & 31 & IPL & 4.57 \\
\hline R postcentral gyrus & 2 & 27 & -37 & 46 & alPS & 4.32 \\
\hline $\mathrm{R}$ middle temporal gyrus & $37 / 39$ & 42 & -58 & 10 & MTG & 3.60 \\
\hline L middle occipital gyrus & 39 & -48 & -70 & 4 & MOG & 4.21 \\
\hline L posterior cingulate & 31 & -15 & -25 & 37 & & 3.83 \\
\hline \multicolumn{7}{|c|}{ (C) INTERACTION BETWEEN LIKING AND PERCEIVED PHYSICAL ABILITY } \\
\hline L middle temporal gyrus & 37 & -54 & -64 & -2 & MTG & 7.51 \\
\hline L inferior occipital gyrus & $37 / 19$ & -45 & -76 & -14 & IOG & 5.10 \\
\hline L inferior occipital gyrus & 19 & -39 & -85 & -8 & IOG & 4.54 \\
\hline R middle temporal gyrus & 19 & 48 & -70 & 1 & V5/MT+ & 7.38 \\
\hline R middle temporal gyrus & 39 & 39 & -58 & 13 & MTG & 5.34 \\
\hline $\mathrm{R}$ middle temporal gyrus & 37 & 54 & -58 & -2 & MTG & 4.86 \\
\hline L posterior cingulate cortex & 31 & -15 & -25 & 37 & & 6.13 \\
\hline R precuneus & 7 & 15 & -52 & 58 & SPL & 3.82 \\
\hline R precuneus & 5 & 9 & -46 & 64 & & 3.79 \\
\hline
\end{tabular}

Locations in MNI coordinates and labels of peaks of relative activation for regions parametrically modulated by increased liking of stimuli (a), decreased physical ability to reproduce the actions observed in the stimuli (b), and the interaction between a and b (c). Results were calculated at $p_{\text {uncorrected }}<0.001, k=10$ voxels. Up to three local maxima are listed when a cluster has multiple peaks more than $8 \mathrm{~mm}$ apart. Entries in bold denote activations significant at the FDR cluster-corrected level of $p<0.05$. Only regions that reached cluster-corrected significance are illustrated in the figures in the main text. Abbreviations for brain regions: BA, Brodmann's area; $R$, right; L, left; V5/MT+, visuotopic area MT; ITG, inferior temporal gyrus; MTG, middle temporal gyrus; MOG, middle occipital gyrus; IPL, inferior parietal lobule; (a)IPS, (anterior) intraparietal sulcus; V3, third visual complex; SPL, superior parietal lobule.

element to the relationship between liking and action perception: namely, that observers also tend to rate actions that are beyond their physical abilities as more likeable.

At this stage, of course, it is unclear how reliable the relationship is between liking and lack of physical ability. One possible way to further evaluate this relationship would be to implement a training paradigm where participants first observe and rate a range of complex movements as novices, and then train over several days or weeks to attain physical mastery of the movements before observing and rating the same movements again. Such an approach might enable much more precise quantification of how the relationship between liking and physical ability is manifest behaviorally.

\section{NEURAL CORRELATES OF OBSERVING DIFFICULT AND LIKEABLE ACTIONS}

Turning our focus to the imaging data, the most illuminating contrast is the interaction between liking and perceived reproducibility. This interaction analysis revealed brain regions that showed a stronger response the more participants liked watching 
a movement and the less well they thought they could reproduce the same movement. The three main clusters to emerge from this contrast were found in bilateral occipitotemporal cortices and the right IPL. In line with the theory proposed by Freedberg and Gallese (2007), one possible way to interpret the IPL finding is that activation in this region is related to increased "embodied simulation" of movements that we like watching. IPL has been previously implicated in embodied simulation processes by a number of studies (e.g., Keysers et al., 2004; Ebisch et al., 2008), and its association with action perception and performance is further reinforced by the identification of so-called "mirror neurons" in the homologous cortical region of non-human primates (Rizzolatti et al., 2001, 2006; Fogassi and Luppino, 2005). Moreover, recent neuroimaging work with humans provides evidence that neurons within human IPL code action perception and execution in a similar manner (Chong et al., 2008; Oosterhof et al., 2010; for a review, see Rizzolatti and Sinigaglia, 2010).

Thus, it could be that when we generally like watching an action that we cannot physically perform, this part of the cortical motor system "works harder" to try and embody it. Put another way, activity within this portion of sensorimotor cortex may be reflecting an attempt to incorporate physically difficult but visually enjoyable actions into the observer's motor system (for more in-depth discussion of this possibility, see Cross et al., in press-a). Alternatively, this relationship could work in the inverse manner, such that increased activation of IPL when watching physically difficult movements leads to increased liking. Although future experimentation is required to confirm or refute the notion that IPL plays a causal role in embodiment and esthetic evaluations when watching dance (and the direction of this relationship), the evidence we present here adds tentative support to Freedberg and Gallese's (2007) proposal that using one's own body to simulate what is seen in art is related to one's esthetic experience of that art.

Our finding of bilateral occipitotemporal cortices when participants watch actions they like but cannot perform is informed by a recent study on the role of the extrastriate body area (EBA) ${ }^{2}$ in esthetic evaluation (Calvo-Merino et al., 2010). Using transcranial magnetic stimulation (TMS), Calvo-Merino et al. (2010) demonstrated that TMS to premotor cortex enhances participants' performance on an esthetic sensitivity task, while TMS to EBA led to decreased esthetic sensitivity. The authors interpret their finding in terms of a dual-route model of body processing (Urgesi et al., 2007a), wherein representations of body parts (mediated by EBA: see Taylor et al., 2007; Cross et al., 2010), and global whole-body

\footnotetext{
${ }^{2}$ Extrastriate body area, located within the occipitotemporal region of the $\mathrm{AON}$, is a cortical region specialized for perception of human bodies (Downing et al., 2001; Peelen and Downing, 2007). The portion of EBA stimulated by Calvo-Merino et al. (2010) is likely subsumed in the bilateral occipitotemporal clusters reported in the interaction between liking and reproducibility in the present study, in that stimulation foci for EBA in Calvo-Merino et al. (2010) are $5.39 \mathrm{~mm}$ from the maximum of the right middle temporal cluster and $10.19 \mathrm{~mm}$ from the maximum of the left middle temporal cluster found in the present study. Nonetheless, we also advise caution in the interpretation of any of our occipitotemporal activations as "extrastriate body area," due to the fact we did not functionally localize these regions (see Downing et al., 2001; Peelen and Downing, 2007 for discussion of EBA localization). It should also be noted that these clusters span much more of occipitotemporal cortex than just EBA, as anatomical localizations reveal that other (sub)peaks within these clusters fall within motion-responsive extrastriate area V5/MT+ (see Table 2).
}

configurations (mediated by the premotor cortex: see Urgesi et al., 2007b) are evaluated in a complementary manner and integrated to arrive at a decision about the esthetic quality of a stimulus.

The purported involvement of EBA in assigning an esthetic value to bodies is perhaps even more intriguing in light of this region' simplification in representing not only observed bodies, but also the observer's body (David et al., 2007). As David et al. (2007) discuss, one possible process EBA may contribute to is a comparison between one's own body and an observed body. Data from perceiving contortionists (Cross et al., 2010), robotic actions (Cross et al., in press-a), gymnasts (Cross et al., in press-b), and now ballet dancers (present study, parametric effect of physical ability; Figure 3B; Table 2B) are consistent with the notion that the more unlike the observer's body/motor repertoire an observed body/movement is, the greater the response within EBA. The novel contribution from the present study, then, is that such occipitotemporal activity when observing others' bodies might be associated with several, possibly related, processes, including coding the degree of deviation between the observed, and observer's body/physical abilities, the degree of liking, and the interaction between these two factors. At this stage, future work is needed to establish whether any causal relationships exist between these processes.

\section{RELATION OF PRESENT FINDINGS TO PREVIOUS LITERATURE}

Unlike our previous work on action observation and the observer's perceived performance ability (Cross et al., 2006, 2009b), in the present study we found no relationship between AON activity and increasing perceived performance ability. We believe this is most likely due to the fact that participants in the present study had no physical experience with the movements they observed. Prior evidence supports the notion that a lack of physical experience specific to the skills required for performing an observed action leads to only weak AON activity during observation of that action (as was seen in dance novices who observed expert ballet or capoeira movements; Calvo-Merino et al., 2005). We suggest that it would be useful for future work to include a larger range of dance movements or simple actions (such as jumping jacks) when studying the relationship between liking and doing, in order to identify how near to an observer's prior motor experience an observed action needs to be in order to demonstrate increased AON activity for increased perceived performance ability.

In relation to prior research on dance neuroesethetics (CalvoMerino et al., 2008), our findings provide a counterpoint on the role of the AON in esthetic evaluation. While Calvo-Merino et al. (2008) showed participants' group esthetic ratings to be correlated with activity within primary visual cortices and the premotor cortex, when we looked at individual esthetic ratings, we found stronger activation within bilateral occipitotemporal cortices and right IPL. These differences are likely attributable (at least to some degree) to differences in task and analysis strategy. It is also worth noting that Calvo-Merino et al. (2008) found that participants rated movements with a higher level of visual motion as more likeable. In the present study, when we assessed the relationship between group-averaged liking ratings and visual motion (motion energy), we also found a positive linear relationship between these variables, computed as a goodness of fit 
statistical correlation $\left(R^{2}=0.376, p=0.002\right)$. However, unlike Calvo-Merino et al. (2008), we explicitly modeled out differences in visual motion between stimuli, and therefore these differences alone cannot account for visual activations reported in the present study. Nonetheless, on a behavioral level, a positive correlation between visual motion and liking ratings suggests that this relationship could be a productive direction for future investigation.

Another feature of the present findings worth considering is the broader pattern of activity that emerged in the interaction between liking and perceived ability (Table 2C). When using the same statistical threshold as Calvo-Merino et al., 2008; $p_{\mathrm{u}}<0.001$ ), more widespread activation of the AON is seen, including right premotor cortex. The fact that right premotor cortex was involved in esthetic processing in the present study lends additional support to the notion that the premotor portion of the $\mathrm{AON}$ is involved in processing the global features of bodies in action, and this information is also used when assigning an esthetic value such bodies (Urgesi et al., 2007a; Calvo-Merino et al., 2010).

\section{IMPLICATIONS AND FUTURE DIRECTIONS}

Taken together, the present findings provide a useful point of departure for further investigation into the relationship between an observer's physical experience and esthetic evaluation of dance. We suggest that future work in this area has the potential to inform not only scientists about how the brain perceives and appreciates art, but also stands to benefit the dance community (Hagendoorn, 2004, 2010; Cross and Ticini, 2011). One intriguing possibility would be for choreographers to experiment with dimensions of

\section{REFERENCES}

Aglioti, S. M., Cesari, P., Romani, M., and Urgesi, C. (2008). Action anticipation and motor resonance in elite basketball players. Nat. Neurosci. 11, 1109-1116.

Berlyne, D. E. (1974). Studies in The New Experimental Aesthetics: Steps Toward an Objective Psychology of Aesthetic Appreciation. Washington: Hemisphere Co.

Bläsing, B., Puttke, M., and Schack, T. (eds). (2010). The Neurocognition of Dance. London: Psychology Press.

Blood, A. J., and Zatorre, R. J. (2001). Intensely pleasurable responses to music correlate with activity in brain regions implicated in reward and emotion. Proc. Natl. Acad. Sci. U.S.A. 98, 11818-11823.

Bobick, A. F. (1997). Movement, activity and action: the role of knowledge in the perception of motion. Philos. Trans. R. Soc. Lond. B Biol. Sci. 352, 1257-1265.

Buccino, G., Binkofski, F., Fink, G. R., Fadiga, L., Fogassi, L., Gallese, V., Seitz, R. J., Zilles, K., Rizzolatti, G., and Freund, H. J. (2001). Action observation activates premotor and parietal areas in a somatotopic manner: an fMRI study. Eur. J. Neurosci. 13, 400-404.
Calvo-Merino, B., Glaser, D. E., Grezes, J., Passingham, R. E., and Haggard, P. (2005). Action observation and acquired motor skills: an FMRI study with expert dancers. Cereb. Cortex 15, 1243-1249.

Calvo-Merino, B., Grezes, J., Glaser, D. E., Passingham, R. E., and Haggard, P. (2006). Seeing or doing? Influence of visual and motor familiarity in action observation. Curr. Biol. 16, 1905-1910.

Calvo-Merino, B., Jola, C., Glaser, D. E., and Haggard, P. (2008). Towards a sensorimotor aesthetics of performing art. Conscious. Cogn. 17, 911-922.

Calvo-Merino, B., Urgesi, C., Orgs, G., Aglioti, S. M., and Haggard, P. (2010). Extrastriate body area underlies aesthetic evaluation of body stimuli. Exp. Brain Res. 204, 447-456.

Casile, A., and Giese, M. A. (2006). Nonvisual motor training influences biological motion perception. Curr. Biol. 16, 69-74.

Caspers, S., Zilles, K., Laird, A. R., and Eickhoff, S. B. (2010). ALE metaanalysis of action observation and imitation in the human brain. $\mathrm{Neu}$ roimage 50, 1148-1167.

Cela-Conde, C. J., Marty, G., Maestu, F., Ortiz, T., Munar, E., Fernandez, A.,

movement difficulty or complexity and esthetic quality, to determine what features of very simple movements might also result in high esthetic evaluation by observers. Along these lines, if future work establishes a more causal relationship between AON activity levels and esthetic enjoyment, then brain imaging can help to determine whether movements perceived as more difficult reliably result in greater activation of the $\mathrm{AON}$, or whether much simpler movements performed with a particular movement quality can also lead to strong AON activation in the observer, as well as high liking ratings. We also recommend more in-depth investigation into the constituent roles played by different $\mathrm{AON}$ regions (namely premotor, parietal, and occipitotemporal cortices) in esthetic evaluation of dance. As we have discussed previously (Cross and Ticini, 2011), many other avenues for investigating how we perceive and evaluate the performing arts await exploration. The findings from the present study highlight the complexity of quantifying esthetic experience of the performing arts at brain and behavioral levels, as esthetic experience can be influenced by any number of other factors, including the observer's physical ability. Investigating other factors that influence esthetic experience, and how they might interact, offers rich opportunities for future studies.

\section{ACKNOWLEDGMENTS}

The authors would like to thank Julia Lechinger for assistance with data collection, Richard Ramsey for helpful comments on an earlier draft of the manuscript, Lauren R. Alpert with manuscript preparation, and the Leipziger Ballett for assistance with stimulus generation.

Roca, M., Rossello, J., and Quesney, F. (2004). Activation of the prefrontal cortex in the human visual aesthetic perception. Proc. Natl. Acad. Sci. U.S.A. 101, 6321-6325.

Chatterjee, A. (2011). Neuroaesthetics: a coming of age story. J. Cogn. Neurosci. 23, 53-62.

Chong, T. T., Cunnington, R., Williams, M. A., Kanwisher, N., and Mattingley, J. B. (2008). fMRI adaptation reveals mirror neurons in human inferior parietal cortex. Curr. Biol. 18, 1576-1580.

Cross, E., and Ticini, L. (2011). Neuroaesthetics and beyond: new horizons in applying the science of the brain to the art of dance. Phenomenol. Cogn. Sci. 1-12. doi: 10.1007/s11097-010-9190-y

Cross, E. S., Hamilton, A. F., and Grafton, S. T. (2006). Building a motor simulation de novo: observation of dance by dancers. Neuroimage 31, 1257-1267.

Cross, E. S., Hamilton, A. F., Kraemer, D. J., Kelley, W. M., and Grafton, S. T. (2009a). Dissociable substrates for body motion and physical experience in the human action observation network. Eur. J. Neurosci. 30, 1383-1392.

Cross, E. S., Kraemer, D. J., Hamilton, A. F., Kelley, W. M., and
Grafton, S. T. (2009b). Sensitivity of the action observation network to physical and observational learning. Cereb. Cortex 19, 315-326.

Cross, E. S., Liepelt, R., Hamilton, A. F., Parkinson, J., Ramsey, R., Stadler, W., and Prinz, W. (in press-a). Robotic actions preferentially engage the action observation network. Hum. Brain Mapp. doi: 10.1002/hbm.21361. [Epub ahead of print].

Cross, E. S., Stadler, W., Parkinson, J., Schütz-Bosbach, S., and Prinz, W. (in press-b). The influence of perceptual training on the prediction of complex biological and non-biological action sequences. Hum. Brain Mapp. doi: 10.1002/hbm.21450. [Epub ahead of print].

Cross, E. S., Mackie, E. C., Wolford, G., and Hamilton, A. F. d. C. (2010). Contorted and ordinary body postures in the human brain. Exp. Brain Res. 204, 397-407.

David, N., Cohen, M. X., Newen, A., Bewernick, B. H., Shah, N. J., Fink, G. R., and Vogeley, Kc. (2007). The extrastriate cortex distinguishes between the consequences of one's own and others' behavior. Neuroimage 36, 1004-1014. 
Decety, J., and Grezes, J. (1999). Neural mechanisms subserving the perception of human actions. Trends Cogn. Sci. (Regul. Ed.) 3, 172-178.

Di Dio, C., and Gallese, V. (2009). Neuroaesthetics: a review. Curr. Opin. Neurobiol. 19, 682-687.

Downing, P. E., Jiang, Y., Shuman, M., and Kanwisher, N. (2001). A cortical area selective for visual processing of the human body. Science 293, 2470-2473.

Ebisch, S. J., Perrucci, M. G., Ferretti, A., Del Gratta, C., Romani, G. L., and Gallese, V. (2008). The sense of touch: embodied simulation in a visuotactile mirroring mechanism for observed animate or inanimate touch. J. Cogn. Neurosci. 20, 1611-1623.

Eickhoff, S. B., Heim, S., Zilles, K., and Amunts, K. (2006). Testing anatomically specified hypotheses infunctional imaging using cytoarchitectonic maps. Neuroimage 32, 570-582.

Eickhoff, S. B., Paus, T., Caspers, S., Grosbras, M. H., Evans, A., Zilles, K., and Amunts, K. (2007). Assignmentof functional activations to probabilistic cytoarchitectonic areas revisited. Neuroimage 36, 511-521.

Eickhoff, S. B., Stephan, K. E., Mohlberg, H., Grefkes, C., Fink, G. R., Amunts, K., and Zilles, K. (2005). A newSPM toolbox for combining probabilistic cytoarchitectonic maps and functional imaging data. Neuroimage 25 , 1325-1335.

Fadiga, L., Fogassi, L., Pavesi, G., and Rizzolatti, G. (1995). Motor facilitation during action observation: a magnetic stimulation study. J. Neurophysiol. 73, 2608-2611.

Fogassi, L., and Luppino, G. (2005). Motor functions of the parietal lobe. Curr. Opin. Neurobiol. 15, 626-631.

Freedberg, D., and Gallese, V. (2007). Motion, emotion and empathy in esthetic experience. Trends Cogn. Sci. (Regul. Ed.) 11, 197-203.
Gallese, V., Keysers, C., and Rizzolatti, G. (2004). A unifying view of the basis of social cognition. Trends Cogn. Sci. (Regul. Ed.) 8, 396-403.

Gazzola, V., and Keysers, C. (2009). The observation and execution of actions share motor and somatosensory voxels in all tested subjects: single-subject analyses of unsmoothed fMRI data. Cereb. Cortex 19, 1239-1255.

Grafton, S. T., Arbib, M. A., Fadiga, L., and Rizzolatti, G. (1996). Localization of grasp representations in humans by positron emission tomography. 2. Observation compared with imagination. Exp. Brain Res. 112, 103-111.

Grèzes, J., and Decety, J. (2001). Functional anatomy of execution, mental simulation, observation, and verb generation of actions: a metaanalysis. Hum. Brain Mapp. 12, 1-19.

Grosbras, M. H., Beaton, S., and Eickhoff, S. B. (in press). Brain regions involved in human movement perception: a quantitative voxel-based meta-analysis. Hum. Brain Mapp. doi: 10.1002/hbm.21222. [Epub ahead of print].

Hagendoorn, I. G. (2004). Towards a Neurocritique of Dance. BalletTanz Yearbook 62-67.

Hagendoorn, I. G. (2010). "Dance, choreography, and the brain," in Art and the Senses, eds D. Melcher and F. Bacci (Oxford: Oxford University Press), 499-514.

Hayes, A. E., Paul, M. A., Beuger, B., and Tipper, S. P. (2008). Self produced and observed actions influence emotion: the roles of action fluency and eye gaze. Psychol. Res. 72, 461-472.

Jacoby, L. L., and Dallas, M. (1981). On the relationship between autobiographical memory and perceptual learning. J. Exp. Psychol. Gen. 110, 306-340.

Kawabata, H., and Zeki, S. (2004). Neural correlates of beauty. J. Neurophysiol. 91, 1699-1705.
Keysers, C., Wicker, B., Gazzola, V. Anton, J. L., Fogassi, L., and Gallese, V. (2004). A touching sight: SII/PV activation during the observation and experience of touch. Neuron 42 , 335-346.

Molenberghs, P., Cunnington, R., and Mattingley, J. B. (in press). Brain regions with mirror properties: a meta-analysis of 125 human fMRI studies. Neurosci. Biobehav. Rev. PMID: 21782846. [Epub ahead of print].

Oldfield, R. C. (1971). The assessment and analysis of handedness: the Edinburgh inventory. Neuropsychologia 9, 97-113.

Oosterhof, N. N., Wiggett, A. J., Diedrichsen, J., Tipper, S. P., and Downing, P. E. (2010). Surfacebased information mapping reveals crossmodal vision-action representations in human parietal and occipitotemporal cortex. J. Neurophysiol. 104, 1077-1089.

Peelen, M. V., and Downing, P. E. (2007). The neural basis of visual body perception. Nat. Rev. Neurosci. 8, 636-648.

Rizzolatti, G., Ferrari, P. F., Rozzi, S., and Fogassi, L. (2006): The inferior parietal lobule: where action becomes perception. Novartis Found. Symp. 270, 129-169.

Rizzolatti, G., Fogassi, L., and Gallese, V. (2001). Neurophysiological mechanisms underlying the understanding and imitation of action. Nat. Rev. Neurosci. 2, 661-670.

Rizzolatti, G., and Sinigaglia, C. (2010). The functional role of the parietofrontal mirror circuit: interpretations and misinterpretations. Nat. Rev. Neurosci. 11, 264-274.

Schippers, M. B., Roebroeck, A., Renken, R., Nanetti, L., and Keysers, C. (2010). Mapping the information flow from one brain to another during gestural communication. Proc. Natl. Acad. Sci. U.S.A. 107, 9388-9393.
Taylor, J. C., Wiggett, A. J., and Downing, P. E. (2007). Functional MRI analysis of body and body part representations in the extrastriate and fusiform body areas. J. Neurophysiol. 98, 1626-1633.

Urgesi, C., Calvo-Merino, B., Haggard, P., and Aglioti, S. M. (2007a). Transcranial magnetic stimulation reveals two cortical pathways for visual body processing. J. Neurosci. 27, 8023-8030.

Urgesi, C., Candidi, M., Ionta, S., and Aglioti, S. M. (2007b). Representation of body identity and body actions in extrastriate body area and ventral premotor cortex. Nat. Neurosci. 10, 30-31.

Whittlesea, B. W. A. (1993). Illusions of familiarity. J. Exp. Psychol. Learn. Mem. Cogn. 19, 12235-12253.

Zeki, S. (1999). Art and the brain. J. Conscious. Stud. 6, 76-96.

Conflict of Interest Statement: The authors declare that the research was conducted in the absence of any commercial or financial relationships that could be construed as a potential conflict of interest.

Received: 03 May 2011; paper pending published: 15 June 2011; accepted: 03 September 2011; published online: 21 September 2011.

Citation: Cross ES, Kirsch L, Ticini LF and Schütz-Bosbach S (2011) The impact of aesthetic evaluation and physical ability on dance perception. Front. Hum. Neurosci. 5:102. doi: 10.3389/fnhum.2011.00102

Copyright (๑) 2011 Cross, Kirsch, Ticini and Schütz-Bosbach. This is an openaccess article subject to a non-exclusive license between the authors and Frontiers Media SA, which permits use, distribution and reproduction in other forums, provided the original authors and source are credited and other Frontiers conditions are complied with. 